\title{
Tratamiento de leucemia linfoide aguda en recaída o enfermedad persistente en un centro de referencia nacional de oncología pediátrica con esquema clofarabina, ciclofosfamida, etopósido
}

- Agustín Darío Contreras Acosta, Teresa Adriana Linares Ballesteros, Nelson Hernando Aponte Barrios, Édgar Vladimir Cabrera Bernal, Carlos Alberto Pardo González, Jymmy Javier Lagos Ibarra, Jhonny Francisco García Medina

Introducción: la recaída en pacientes con leucemia linfoblástica aguda (LLA) es de difícil manejo y con posibilidad de sobrevida muy baja, por lo que es necesario evaluar nuevos protocolos de rescate que permitan mejorar el pronóstico de estos pacientes.

Objetivo: describir la respuesta de remisión completa $(\mathrm{RC})$ después de al menos un ciclo de terapia combinada con clofarabina, etopósido y ciclofosfamida para tratamiento de pacientes pediátricos con LLA con enfermedad persistente o en recaída.

Materiales y métodos: pacientes y métodos: se realizó un estudio de cohorte descriptiva; entre abril de 2012 y diciembre de 2017, se diagnosticaron en la Fundación HOMI Hospital de la Misericordia 20 pacientes menores de 18 años con LLA refractaria al tratamiento inicial o en recaída que fueron tratados con combinación de clofarabina $40 \mathrm{mg} / \mathrm{m}^{2} /$ día, ciclofosfamida $400 \mathrm{mg} / \mathrm{m}^{2} /$ día y etopósido $150 \mathrm{mg} / \mathrm{m}^{2} /$ día por cinco días, con profilaxis antimicótica, antibacteriana y antiviral, más soporte transfusional intensivo.

Resultados: de los 20 pacientes, 7 pacientes entraron en remisión completa posterior a primer ciclo, permitiendo realizar en ellos trasplante de progenitores hematopoyéticos, con toxicidad semejante a otros protocolos de menor intensidad.

Conclusiones: la combinación de clofarabina, citarabina y etopósido se constituye como una posibilidad de tratamiento para los pacientes con enfermedad refractaria o recaída en pacientes con LLA en la búsqueda de remisión completa para poder ser llevados a consolidación con trasplante alogénico de progenitores hematopoyéticos. 